\title{
Overexpression of Rab40b Promotes Hepatocellular Carcinoma Cell Proliferation and Metastasis via PI3K/AKT Signaling Pathway
}

This article was published in the following Dove Press journal: Cancer Management and Research

\author{
Li-Wei Shi ${ }^{1,2}$ \\ Zhi-Bo Zhao ${ }^{3}$ \\ Li Zhong' \\ Jian Gao' \\ Jian-Ping Gong ${ }^{3}$ \\ Hang Chen ${ }^{4}$ \\ Yu Min ${ }^{4}$ \\ Yi-Yin Zhang ${ }^{5}$ \\ Zhi Li ${ }^{4}$
}

'Department of Gastroenterology, The Second Affiliated Hospital of Chongqing Medical University, Chongqing 400010 ,

People's Republic of China; ${ }^{2}$ Department of Gastroenterology, Chongqing People's Hospital, Chongqing 400010, People's Republic of China; ${ }^{3}$ Department of Hepatobiliary Surgery, The Second Affiliated Hospital of Chongqing Medical University, Chongqing 400010, People's Republic of China; ${ }^{4}$ Department of Breast \& Thyroid Surgery, The Second Affiliated Hospital of Chongqing Medical University, Chongqing 4000 I0, People's Republic of China; ${ }^{5}$ Department of Anesthesiology, Chongqing Hospital of Traditional

Chinese Medicine, Chongqing 40II 20,

People's Republic of China
Correspondence: Zhi Li

Department of Breast \& Thyroid Surgery, The Second Affiliated Hospital of

Chongqing Medical University, Yuzhong

District, Chongqing 4000 I0, People's

Republic of China

$\mathrm{Tel}+86$ I8883I47602

Email 393884358@qq.com
Background: Rab40b is an evolutionarily conserved Rab GTPase that plays an important role in intracellular trafficking and is closely related to cancer progression. However, the role and potential molecular mechanism of Rab40b in hepatocellular carcinoma (HCC) have not yet been elucidated.

Materials and Methods: The expression of Rab40b in HCC tissues and peritumour tissues was tested by qRT-PCR, Western blotting and histological analysis. A Kaplan-Meier survival curve was generated based on the expression of Rab40b in the HCC samples. Cell proliferation assays, wound healing assays, and transwell assays are used to examine the effect of Rab40b on HCC cell growth in vitro. The effect of Rab40b on cell cycle was examined by flow cytometry. A xenograft implantation model was used to assess the effect of Rab40b on the development of HCC cells in vivo.

Results: Rab40b protein expression is upregulated in HCC tissues, and this upregulation is associated with high pathological stage and poor prognosis in HCC patients. Rab40b overexpression promotes the proliferation and metastasis of HCC cells by upregulating cyclin D1, cyclin E1 and matrix metalloproteinase 2 (MMP2) through the PI3K/AKT signalling pathway. Conversely, Rab40b inhibitors can significantly inhibit the proliferation and metastasis of HCC cell lines and induce G0/G1 cell cycle arrest and apoptosis. Studies of a nude mouse xenograft model demonstrated that Rab40b knockdown can significantly inhibit the proliferation and progression of HCC tumours in vivo.

Conclusion: Overall, this study demonstrates that Rab40b is an important oncoprotein that promotes HCC progression by regulating the expression of cyclin D1, cyclin E1, p21 and MMP2 through the PI3K/AKT signalling pathway.

Keywords: Rab40b, HCC, cell cycle, PI3K/AKT, cyclins, MMP2

\section{Introduction}

Hepatocellular carcinoma (HCC) is a malignant tumour that seriously endangers human life, ranking third in terms of global malignant tumour mortality rate. ${ }^{1}$ In recent years, numerous studies have provided insights into the diagnosis and treatment of HCC. However, the molecular mechanism of HCC pathogenesis is still poorly understood. ${ }^{2}$

Growing evidence has shown that Rab protein plays an important role in the progression of HCC. ${ }^{3}$ Rab proteins are a class of small GTPases belonging to the Ras protein family and can be used as regulators of protein transport, vesicle transport, membrane targeting and fusion. Modification of the mutated Rab protein 
leads to an imbalance in the intracellular material transport network, a process that is critical in tumorigenesis. ${ }^{4-6}$ Recently, some Rab GTPases have been shown to be involved in the progression of HCC. For example, RAB10 is overexpressed in HCC tumours and promotes the proliferation of HCC cells. ${ }^{7}$ In addition, overexpression of RAB25 is associated with advanced tumour staging and lymph node metastasis in HCC progression. ${ }^{8}$ Rab40b is a member of the Rab GTPase family protein. ${ }^{9}$ Recent studies have found that Rab40b is involved in the progression of several cancers. ${ }^{10}$ It has been reported that Rab40b is upregulated in gastric cancer and affects the prognosis of gastric cancer patients. ${ }^{11}$ Some studies have reported that Rab40b regulates the formation of invadopodia and participates in the process of breast cancer cell metastasis by mediating the transport of MMP2 and MMP9. ${ }^{12}$ However, the regulatory role and mechanism of Rab40b in HCC have not been elucidated.

In this study, we demonstrated that Rab40b protein is significantly upregulated in HCC tissues and is associated with clinical stage and poor prognosis in HCC patients. We found that overexpression of Rab40b promotes the proliferation, invasion and migration of HCC cell lines and regulates the expression of cyclin D1, cyclin E1, p21 and MMP2 through the PI3K/AKT signalling pathway. The Rab40b inhibitor induced G0/G1 cell cycle arrest and apoptosis in HCC cells. Therefore, this study shows that Rab40b is an important oncoprotein and a promising target in HCC therapy. Our results may provide new potential therapeutic targets for the treatment of advanced HCC.

\section{Materials and Methods}

\section{Cell Lines and Cell Cultures}

Hepatocellular carcinoma cell lines HepG2, SK-Hep1 were purchased from the American Type Culture Collection (ATCC; Manassas, VA, USA). Hepatocellular carcinoma cell lines Hep3B, Huh7, SMMC-7721 and the normal hepatocyte cell line MIHA were purchased from the Cell Bank of the Chinese Academy of Sciences (Shanghai, China). Hepatocellular carcinoma cell lines were all cultured in Dulbecco's modified Eagle's medium (DMEM, HyClone, Logan, UT, USA) containing $10 \%$ FBS and $1 \%$ penicillin $\mathrm{G}$ and incubated in humidified air containing $5 \% \mathrm{CO} 2$ at $37^{\circ} \mathrm{C}$.

\section{Human Subjects}

Tissue microarray analysis was performed on 90 HCC patients who underwent radical HCC surgery from 2006 to 2009 (see online Supplementary Table S1). None of the patients received chemotherapy or radiotherapy before surgery. The patients were followed up for 4-6 years according to their postoperative survival. Informed consent was signed by all patients for the retention and use of their tissues in scientific research only. This project was approved by the Human Research Ethics Committee of the Second Affiliated Hospital of Chongqing Medical University.

\section{Immunohistochemistry}

Antibody staining was observed by DAB and haematoxylin counterstaining. The cell-staining rate and staining intensity were evaluated and averaged by 2 experienced pathologists. The staining intensity was scored as follows: 0 indicates no staining, 1 indicates weak staining, 2 indicates intermediate staining, and 3 indicates strong staining. The positive rate was scored from 0 to 5: $0(0 \%), 1(1 \%-20 \%), 2(21-40 \%)$, $3(41-60 \%), 4(61-80 \%)$, and $5(81-100 \%)$. The cell-staining rate and staining intensity were multiplied to determine the total score. A total score $<6$ indicates low expression, and a score $\geq 6$ indicates high expression.

\section{Cell Proliferation Assay}

Cell proliferation was measured using the Cell Counting Kit8 (Dojindo, Tokyo, Japan) assay following the manufacturer's protocol. Approximately $1 \times 10^{3}$ cells were seeded into each well of 96-well plates, and cell proliferation was assessed every 24 hours after incubation with $10 \mu \mathrm{L}$ CCK-8 solution per well for another 2 hours. The absorbance at 450 $\mathrm{nm}$ was measured by the enzyme marker, and the growth curve was plotted over 6 days.

\section{Wound Healing Assays}

Cells were seeded in six-well plates and incubated until they reached complete confluence. The confluent monolayers were wounded in a line using a sterile plastic pipette tip and washed with PBS to remove any detached cells. Cell migration distance across the wound was measured over a fixed time interval. For accuracy, at least three regions were selected for statistical analysis.

\section{Transwell Migration Assays}

A total of $200 \mu \mathrm{L}$ serum-free medium containing $1 \times 10^{4}$ cells was added into the upper chamber of a Transwell ${ }^{\mathrm{TM}}$ insert (Millipore, MA, USA), and the 24-well plates were filled with $500 \mu \mathrm{L}$ complete medium. After 24 hours, cells that did not invade through the membrane were removed, 
and migrated cells were fixed with $4 \%$ paraformaldehyde, stained with $0.1 \%$ crystal violet, and counted in three random fields.

\section{Cell Cycle and Apoptosis}

SMMC-7721 and Huh7 cells infected with Lv-shRab40b or Lv-Rab40b were detected to determine the apoptosis rate and the proportion of cells in different cell cycle phases by flow cytometry (FACScan ${ }^{\circledR}$; BD Biosciences) following the manufacturer's protocol. The results were quantified and compared to those of the shCont.

\section{In vivo Tumourigenicity}

Four-week-old male BALB/c nude mice were purchased from the animal institution of the Chinese Academy of Medical Science. A total of $1 \times 10^{7} \mathrm{HCC}$ cells transfected with Lv-Rab40b or Lv-NC (Lv-shRab40b or Lv-shCont) were injected subcutaneously into nude mice from the flank of both sides at the dorsal region, and tumour size was measured every 2 days. Tumour volume was calculated using the formula: volume $=1 / 2 \times$ length $\times$ width $^{2}$. After observation for 11 days, the mice were sacrificed, and the tumours were weighed and fixed with paraformaldehyde for immunohistochemistry. The study was approved by the Animal Care and Use Committee of Chongqing Medical University and animal care and use protocols adhered to national regulations for the administration of laboratory animals.

\section{Statistical Analysis}

Quantitative data are presented as the mean \pm standard deviation (SD). Statistical analyses were performed using SPSS (version 22.0). Student's t-tests were used to compare two groups of independent samples. ANOVA was performed to compare multiple groups. Nonparametric tests were used to analyse the difference in Rab40b expression between HCC and adjacent tissues. Spearman correlation analysis was used to analyse the correlation between Rab40b protein expression and clinicopathological parameters. Kaplan-Meier survival analysis and log-rank statistical tests were used for single factor survival analysis. A Cox regression model was used for multivariate survival analysis to identify prognostic factors that were significant in the univariate analysis. $\mathrm{P}<0.05$ was considered statistically significant.

\section{Results}

Rab40b was Significantly Upregulated in HCC Tumour Tissues and Associated with High Pathological Grade and Poor Survival in HCC Patients

To elucidate the function of Rab40b in HCC, a tissue microarray was used to examine the expression of Rab40b in HCC tissues. Immunohistochemical staining showed that Rab40b was significantly upregulated in tumour tissues compared to adjacent tissues (Figure 1A). In addition, Western blotting confirmed that Rab40b was overexpressed in tumour tissues (Figure 1B). As shown in Figure 1C, the results of immunohistochemical staining showed that the expression level of Rab40b protein in the nucleus of HCC tissue was significantly higher than that of adjacent tissues $(5.81 \pm 4.91$ vs 0.63 $\pm 1.69, \mathrm{P}=0.000)$. However, the expression level of Rab40b protein in the cytoplasm of HCC tissues was not significantly different from that in peritumour tissues $(9.32 \pm 3.45$ vs 9.61 $\pm 2.38, \mathrm{P}=0.506)$. In addition, the Oncomine database (https://www.oncomine.org/resource/login.html) was used to analyse the expression levels of Rab40b mRNA in HCC and normal tissues (Figure 1D). Compared with the normal group, the expression level of Rab40b mRNA in HCC tissue was dramatically upregulated $(\mathrm{P}<0.05)$. The conclusion of these results is that Rab40b expression is significantly upregulated in HCC tumour tissues.

The Spearman test was used to analyse the correlation between Rab40b expression and the clinicopathological characteristics of HCC patients (Table 1). Correlation analysis showed that the expression of Rab40b in the nucleus of HCC tissue was positively correlated with the pathological grade, but there was no significant correlation between the cytoplasmic expression of Rab40b in HCC tissue and other clinical and pathological features. Next, Kaplan-Meier analysis showed that the high expression of Rab40b in tumour tissue was significantly associated with the poor prognosis of patients (Figure 1E shows the data of our study, and Figure 1F shows the data from The Human Protein Atlas). In addition, multivariate analysis by Cox regression showed that Rab40b expression was the only independent prognostic factor (Table 2). In addition, the expression of Rab40b was positively correlated with the expression of Ki-67 (Figure $1 \mathrm{G}$ and $\mathrm{H}$ ). These results indicate that $\mathrm{Rab} 40 \mathrm{~b}$ expression is upregulated in $\mathrm{HCC}$ tumour tissues and is closely related to poor 
A

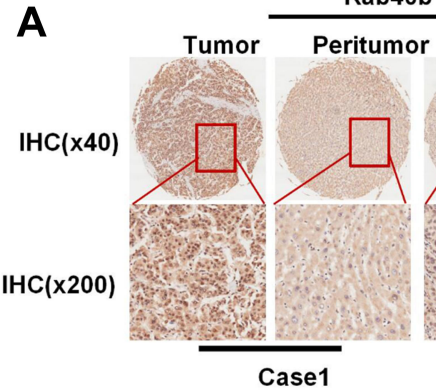

C

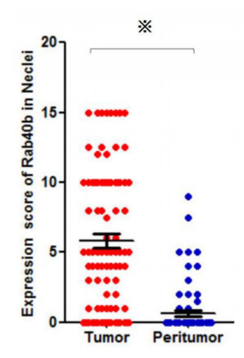

E

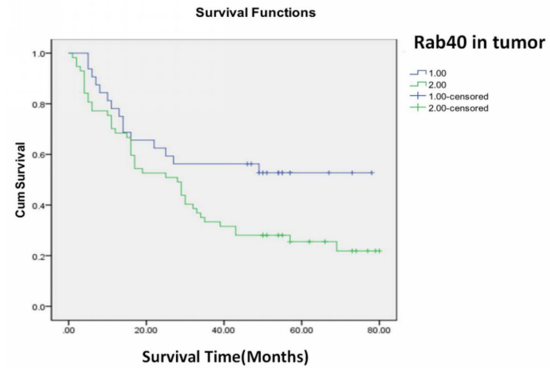

G

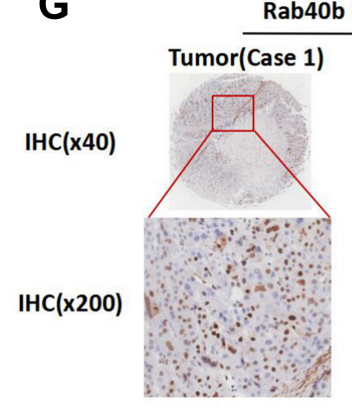

Rab40b in HCC

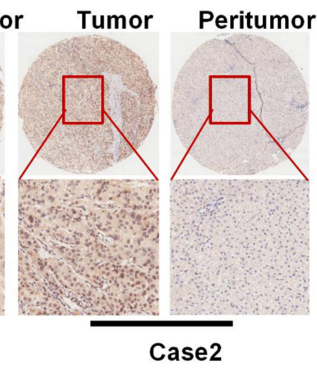

ns

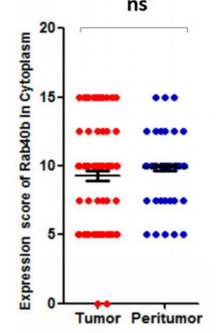

Rab40 in tumor

D

B
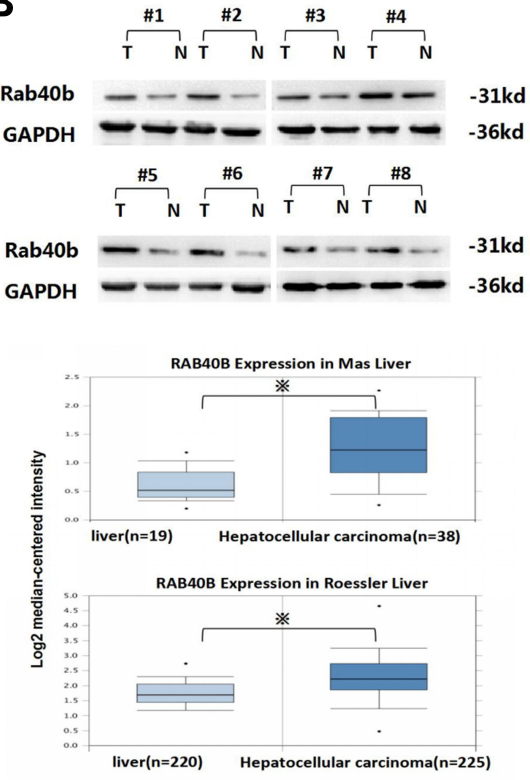

$\mathbf{F}$

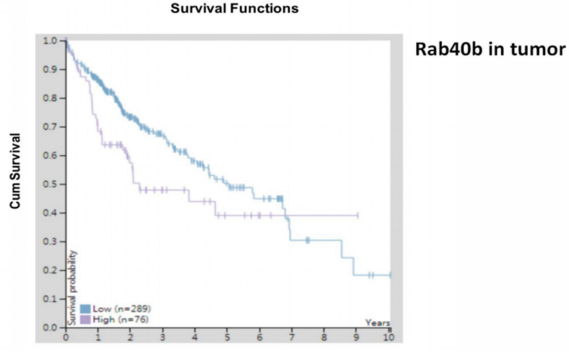

H

Survival Time(Years)

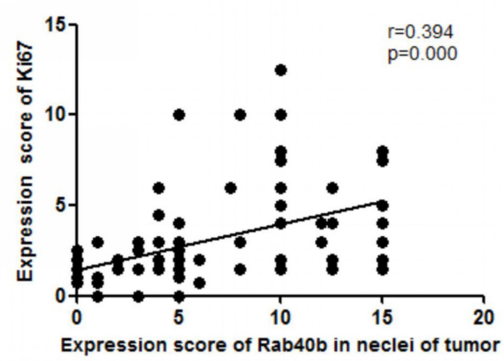

Figure I Rab40b was significantly upregulated in HCC tumour tissues and associated with poor survival in HCC patients. (A) Immunohistochemical staining was utilized to examine Rab40b in HCC tumor tissues and matched peritumor tissues. (magnification at $\times 40$ and $\times 200$ ). (B) Western blot analysis of Rab40b in HCC tissues and matched peritumor tissues. (C) Immunohistochemical scores of Rab40b expression in HCC tumor tissues and peritumor tissues, ${ }^{*} \mathrm{p}<0.05$. (D) Oncomine database mining of Rab40b mRNA level, ${ }^{*} \mathrm{p}<0.05$. (E) The Kaplan-Meier survival curve showed the correlation between Rab40b expression in tumor tissues and survival of HCC patients, I: low Rab40b expression, 2: high Rab40b expression. (F) Kaplan-Meier survival curve on the relationship between Rab40b mRNA expression and OS of HCC patients (data from The Human Protein Atlas). (G) Ki67 expression were tested by immunohistochemical staining in HCC tissues (magnification at $\times 40$ and $\times 200$ ). (H) Rab40b expression was positively correlated with the expression of $\mathrm{Ki67}(\mathrm{p}=0.000, r=0.394)$.

survival of HCC patients. In particular, the expression of Rab40b in the nucleus of HCC tissue is positively correlated with the pathological grade and tumour proliferation of HCC patients, suggesting that Rab40b may mainly play a role in the nucleus of HCC tissues.

\section{Overexpression of Rab40b Significantly Affected HCC Cell Proliferation, \\ Migration and Invasion in vitro}

Our previous results showed that Rab40b expression was significantly correlated with $\mathrm{Ki}-67$ staining and 
Table I The Correlation Between Rab40b Expression and Clinicopathological Characteristics of HCC Patients

\begin{tabular}{|c|c|c|c|c|c|c|c|c|c|c|}
\hline & & & Gender & Age & $\begin{array}{l}\text { Tumor } \\
\text { Size }\end{array}$ & $\begin{array}{l}\text { Differentiated } \\
\text { Grade }\end{array}$ & $\mathrm{T}$ & $\mathrm{N}$ & $M$ & $\begin{array}{l}\text { TNM } \\
\text { Stage }\end{array}$ \\
\hline \multirow[t]{6}{*}{$\begin{array}{l}\text { Spearman's } \\
\text { rho }\end{array}$} & \multirow{3}{*}{$\begin{array}{l}\text { Expression } \\
\text { of Rab } 40 \mathrm{~b} \\
\text { in the nucleus of tumor } \\
\text { tissues }\end{array}$} & $\begin{array}{l}\text { Correlation } \\
\text { Coefficient }\end{array}$ & 0.108 & -.162 & -.017 & 0.285 & 0.066 & -.149 & 0.081 & 0.033 \\
\hline & & Sig. (2-tailed) & 0.314 & 0.131 & 0.876 & $0.007 * *$ & 0.548 & 0.177 & 0.459 & 0.766 \\
\hline & & $\mathrm{N}$ & 89 & 88 & 88 & 89 & 86 & 84 & 85 & 85 \\
\hline & \multirow{3}{*}{$\begin{array}{l}\text { Expression of Rab } 40 \mathrm{~b} \\
\text { in the nucleus of para- } \\
\text { carcinoma tissue }\end{array}$} & $\begin{array}{l}\text { Correlation } \\
\text { Coefficient }\end{array}$ & 0.122 & 0.143 & 0.106 & -.054 & -.021 & -.053 & -.074 & -.016 \\
\hline & & Sig. (2-tailed) & 0.272 & 0.197 & 0.343 & 0.625 & 0.855 & 0.645 & 0.515 & 0.891 \\
\hline & & $N$ & 83 & 83 & 82 & 83 & 80 & 78 & 79 & 79 \\
\hline & \multirow{3}{*}{$\begin{array}{l}\text { Expression } \\
\text { of Rab40b } \\
\text { in cytoplasm of tumor } \\
\text { tissues }\end{array}$} & $\begin{array}{l}\text { Correlation } \\
\text { Coefficient }\end{array}$ & -.188 & 0.163 & -.036 & -.117 & -.025 & 0.030 & -.118 & -.075 \\
\hline & & Sig. (2-tailed) & 0.080 & 0.131 & 0.741 & 0.277 & 0.823 & 0.789 & 0.287 & 0.500 \\
\hline & & $N$ & 88 & 87 & 87 & 88 & 85 & 83 & 84 & 84 \\
\hline & \multirow{3}{*}{$\begin{array}{l}\text { Expression of Rab40b } \\
\text { In cytoplasm of para- } \\
\text { carcinoma tissue }\end{array}$} & $\begin{array}{l}\text { Correlation } \\
\text { Coefficient }\end{array}$ & -.167 & -.053 & 0.111 & 0.034 & 0.110 & 0.016 & -.150 & 0.085 \\
\hline & & Sig. (2-tailed) & 0.129 & 0.630 & 0.318 & 0.760 & 0.328 & 0.889 & 0.183 & 0.455 \\
\hline & & $\mathrm{N}$ & 0.108 & -.162 & -.017 & 0.285 & 0.066 & -.149 & 0.081 & 0.033 \\
\hline
\end{tabular}

Note: ** Correlation is significant at the 0.01 level (2-tailed).

Table 2 Multivariate Analysis by Cox Regression

\begin{tabular}{|c|c|c|c|c|c|c|c|c|}
\hline & \multirow[t]{2}{*}{ B } & \multirow[t]{2}{*}{ SE } & \multirow[t]{2}{*}{ Wald } & \multirow[t]{2}{*}{$d f$} & \multirow[t]{2}{*}{ P-value } & \multirow[t]{2}{*}{$\operatorname{Exp}(B)$} & \multicolumn{2}{|c|}{ 95.0\% Cl for $\operatorname{Exp}(B)$} \\
\hline & & & & & & & Lower & Upper \\
\hline Rab40b expression & 0.667 & 0.319 & 4.388 & 1 & $0.036^{*}$ & 1.949 & 1.044 & 3.639 \\
\hline Tumor size & 0.250 & 0.355 & 0.494 & 1 & 0.482 & 1.284 & 0.640 & 2.575 \\
\hline $\mathrm{T}$ & 0.392 & 0.624 & 0.393 & 1 & 0.530 & 1.480 & 0.435 & 5.031 \\
\hline Clinical stage & 0.242 & 0.561 & 0.185 & I & 0.667 & 1.273 & 0.424 & 3.826 \\
\hline
\end{tabular}

Note: * Correlation is significant at the 0.05 level (2-tailed).

high pathological grade in HCC patients. Therefore, we examined the effect of Rab40b on HCC cell proliferation and migration. First, we detected the expression of Rab40b in the HCC cell line using Western blotting. The results showed that the expression of Rab40b was upregulated in SK-Hep1, SMMC-7721, HepG2, and Hep3B cells but downregulated in Huh7 cells (Figure 2A). Next, we used lentiviral technology to knock down the expression of Rab40b in SMMC7721 cells with high endogenous Rab40b expression and overexpressed Rab40b expression in Huh7 cells with low endogenous Rab40b expression (Figure 2B). The effect of Rab40b on the proliferation of $\mathrm{HCC}$ cells was detected by CCK-8 assay (Figure 2C). The results showed that Rab40b overexpression promoted the growth rate of Huh7 cells, while knocking down Rab40b inhibited the proliferation rate of SMMC-7721 cells. Wound healing and transwell migration assays were used to assess the effect of Rab40b on HCC cell migration. The wound healing test showed that the healing ability of SMMC-7721shRab40b cells was significantly reduced (Figure 2D). Similarly, Transwell analysis showed that Rab40b knockdown significantly reduced the migration ability (Figure 2E) and invasion ability (Figure 2F) of HCC cells. 

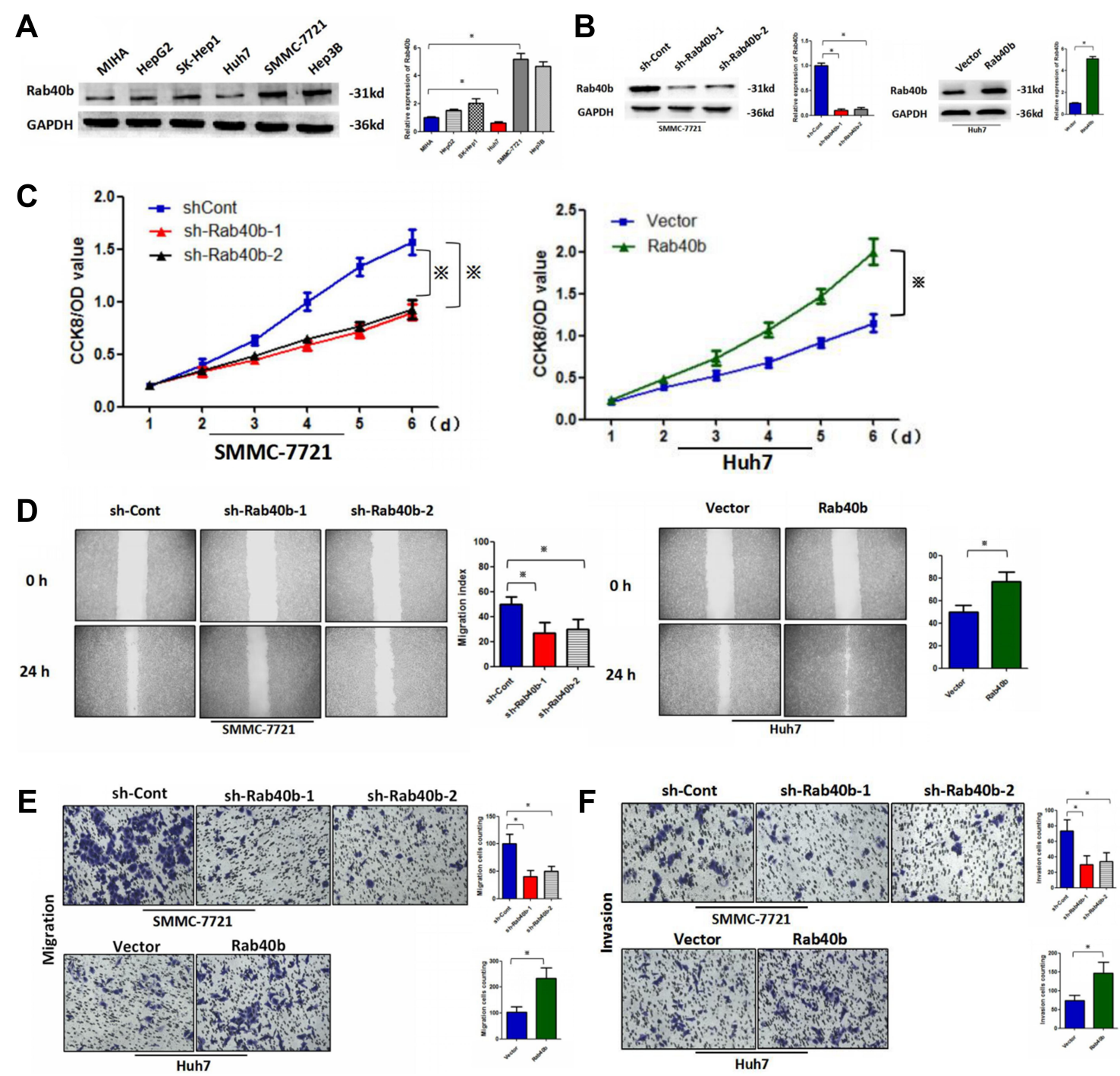

Figure 2 Overexpression of Rab40b significantly affected HCC cell proliferation, migration and invasion in vitro. (A) Expression of Rab40b in hepatocellular carcinoma cell lines, ${ }^{*} p<0.05$. (B) Western blot analysis of Rab40b knockdown efficiency in SMMC-772I and overexpression efficiency in Huh7, ${ }^{*} \mathrm{p}<0.05$. (C) CCK-8 assay showed the effect of Rab40b expression on HCC cell proliferation, ${ }^{*} \mathrm{p}<0.05$. (D) Wound healing assays detected the effect of Rab40b knockdown or overexpression on the healing ability of HCC cells, ${ }^{*} \mathrm{p}<0.05$. (E) Transwell assays evaluated the effect of Rab40b expression on the migratory ability of $\mathrm{HCC}$ cells, ${ }^{*} \mathrm{p}<0.05$. (F) Transwell assays evaluated the effect of Rab40b expression on the invasion ability of $\mathrm{HCC}$ cells, ${ }^{*} \mathrm{p}<0.05$.

\section{Knockdown of Rab40b Induced HCC} Cell Cycle Arrest and Apoptosis by Downregulating Cyclin DI and Cyclin EI and Upregulating P2I via the PI3K/AKT Signalling Pathway

Our previous results suggested that Rab40b expression significantly impacted HCC cell proliferation. However, alterations in cell proliferation are usually caused by changes in the cell cycle or apoptosis. Therefore, we analysed the effect of Rab40b on the cell cycle or apoptosis by flow cytometry. As shown in Figure 3A, the results showed that the shRab40b group displayed a significant arrest in the G0/G1 phase compared with the control group, which suggested that the effect of Rab40b on HCC proliferation was closely related to the cell cycle distribution $(\mathrm{P}<0.05)$. Moreover, as shown in Figure 3B, compared with the control group, the percentage of SMMC-7721 cells in the apoptotic phase of the shRab40b group was significantly higher. These results indicate that Rab40b may be associated with the apoptosis of HCC cells. To investigate the possible mechanism, we compared the transcriptome of shRab40b lentivirusinfected HCC cells and NC lentivirus-infected cells. A volcano plot showed a total of 878 gene expression changes significantly, including 421 upregulated genes 

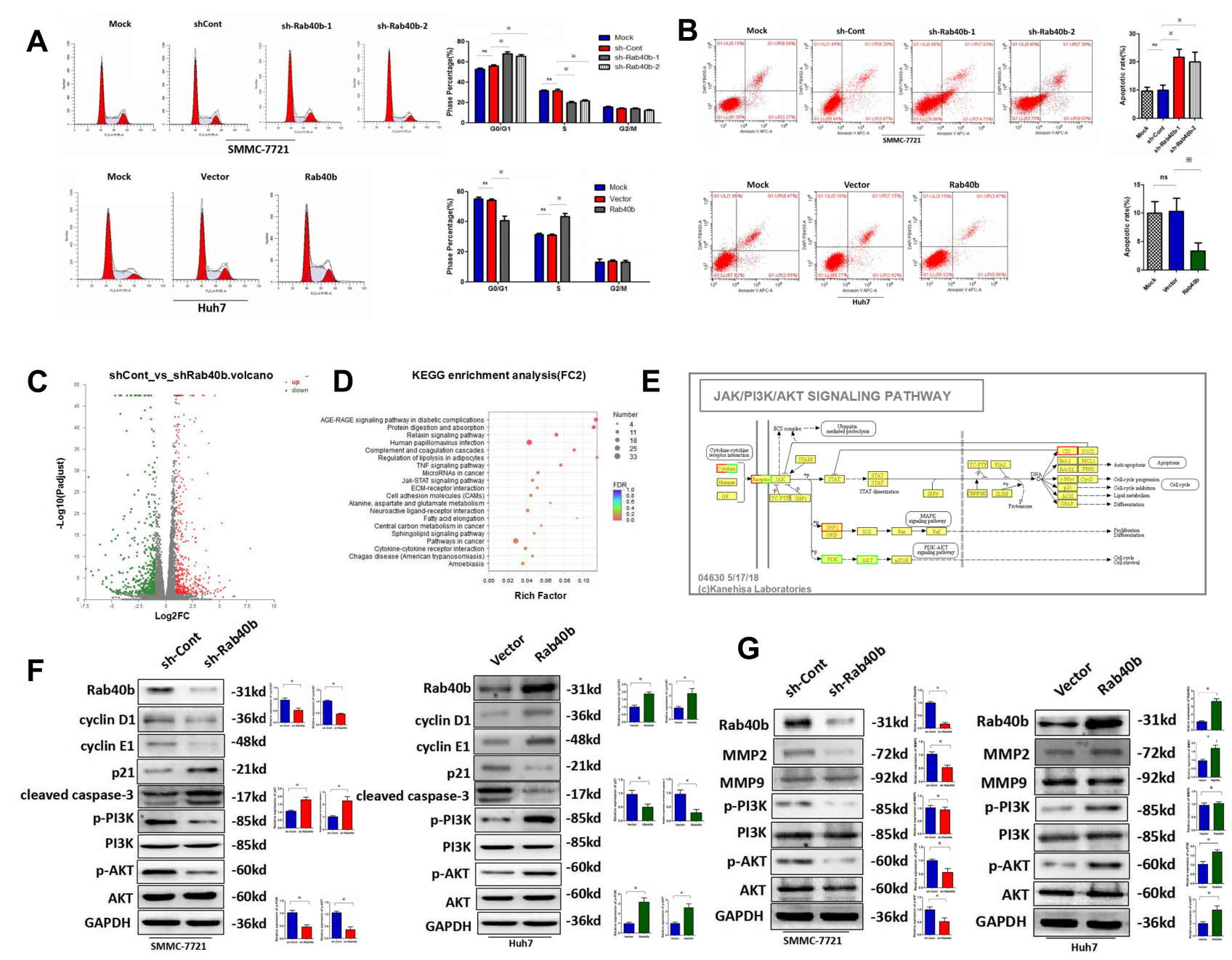

Figure 3 Knockdown of Rab40b induced HCC cell cycle arrest and apoptosis by downregulating cyclin DI and cyclin EI and upregulating P2I via the PI3K/AKT signalling pathway. (A) The percentages of cells in the G0/GI, S, G2/M phases were examined by FCM,*p < 0.05. (B) The apoptosis rate of HCC cells were evaluated by FCM, *p < 0.05. (C) Volcano plot of 878 genes showed significant $(p<0.05)$ differential expression (fold change $>2$ ) between cells transfected with shRab40b and with shControl (green represented down-regulated genes and red represented up-regulated genes). (D) Functional pathway enrichment of differentially expressed genes was analyzed based on KEGG databases. Statistically significant modulations $(\mathrm{p}<0.05)$ of the top 20 pathways were shown. (E) Functional pathway analysis revealed that the PI3K/AKT signaling pathway was the modulated (green represented modulated genes, $\mathrm{P}<0.05$ ). (F) Western blot analysis of PI3K/AKT signaling pathway and several cell cycle-related proteins after knockdown or overexpression of Rab40b, ${ }^{*} \mathrm{p}<0.05$. (G) Western blot analysis of PI3K/AKT signaling pathway and matrix metalloproteinases after knockdown or overexpression of Rab40b, ${ }^{\mathrm{p}}<0.05$.

and 457 downregulated genes with $\mathrm{P}<0.05$ and $>2.0$ absolute value of fold change (Figure 3C). KEGG functional pathway enrichment analysis of differentially expressed genes revealed the top 20 related pathways, as shown in Figure 3D. The pathway analysis showed that PI3K/AKT signalling pathways related to the cell cycle and cell survival were significantly modulated (Figure 3E). In addition, Western blot results showed that Rab40b silencing decreased the levels of p-PI3K and p-Akt (Figure 3F). Furthermore, we compared the expression levels of various cyclins and other cell cycle-activating proteins that are pathway-related or downstream genes of PI3K/AKT signalling pathways. Our results demonstrated that Rab40b knockdown decreased the levels of cyclin D1 and cyclin E1, which closely regulate the cell cycle, and increased the levels of cleaved caspase- 3 and p21, which are associated with cell apoptosis through the PI3K/AKT signalling pathway (Figure 3F). In conclusion, these results indicate that knockdown of Rab40b induced HCC cell cycle arrest and apoptosis by downregulating cyclin D1 and cyclin E1 and upregulating p21 via the PI3K/AKT signalling pathway.

Moreover, in order to explore the mechanism by which Rab40b promotes metastasis of HCC cells. We examined the PI3K/AKT pathway and the protein expression of matrix metalloproteinases, which are closely related to 
tumor metastasis. Our results demonstrated that Rab40b knockdown significantly decreased the levels of MMP2, p-PI3K and p-AKT, while the expression of MMP9 was no changed (Figure 3G). These results indicate that knockdown of Rab40b inhibited metastasis of HCC cells by downregulating MMP2 via the PI3K/AKT signalling pathway.

\section{Overexpression of Rab40b Promoted the Development of Xenografts in BALB/C Mice}

Since Rab40b silencing induced the inhibition of HCC cells by downregulating cyclin D1, cyclin E1 and MMP2, we examined the critical role of Rab40b in HCC tumorigenesis in vivo. A mouse xenograft model was generated to examine the effect of Rab40b knockdown on the growth of HCC cells. As shown in Figure 4A and $\mathrm{B}$, significant repression of tumour growth was observed in nude mice with Rab40b silencing. Similarly, the tumour weight of the Lv-shRab40b group was less than that of the Lv-shControl group (Figure 4C), whereas there is no statistical difference in the body weight of nude mice between each group. Since the inhibition of Rab40b is closely related to cell proliferation and metastasis, the expression of Ki-67, cyclin D1, cyclin E1 and MMP2 was evaluated in tumour tissues. Histological analysis further confirmed that the expression of Ki-67, cyclin D1, cyclin E1 and MMP2 in tumour tissues was significantly reduced with Rab40b knockdown (Figure 4G). Similarly, the Rab40b-overexpressing mouse model supports these results. As shown in Figure 4D-F, the overexpression of Rab40b promoted the growth and development of xenografts. Histological analysis confirmed that the expression of Ki-67, cyclin D1, cyclin E1 and MMP2 was significantly increased in the tumour tissue with Rab40b overexpression (Figure 4H). Taken together, these results suggest that Rab40b silencing inhibits tumour growth and invasiveness in vitro and in vivo.

\section{Discussion}

Hepatocellular carcinoma (HCC) is the most important pathological type of primary liver cancer. ${ }^{13,14}$ Among people over 60 years old, HCC has a high morbidity and mortality rate among all cancers. ${ }^{15,16}$ Rab proteins are a class of small-molecule GTPases. ${ }^{17,18}$ Recently, many Rab GTPases have been confirmed to be involved in the occurrence and development of HCC. ${ }^{19}$ It has been reported that Rab31 can promote the progression of $\mathrm{HCC}$ by inhibiting the apoptosis of HCC cells. ${ }^{20}$ Rab40b is a member of the Rab family, and abnormal expression of Rab40b is found in a variety of cancer types. ${ }^{21,22}$ However, previous studies have focused on the function of Rab40b in transportation, and the biological significance of Rab40b levels in HCC has not been explored.

In this study, we first studied the function of Rab40b expression in HCC tissues. We detected Rab40b expression in $\mathrm{HCC}$ tissues from $90 \mathrm{HCC}$ patients receiving curative liver resection. Immunohistochemical staining showed that the expression of Rab40b in the nucleus of HCC cells was significantly higher than that in peritumour tissues. Abnormally high expression of Rab40b in HCC tissues is related to the high pathological grade and poor prognosis of HCC patients, which is consistent with the correlation of Rab40b protein expression in gastric cancer. ${ }^{11}$ Kaplan-Meier analysis and log-rank statistical test were used for univariate survival analysis. Our results demonstrated that there was no correlation between gender $(p=0.737)$, age $(p=0.349)$, pathological grade $(p=0.598)$, $\mathrm{N}$ staging $(\mathrm{p}=0.272), \mathrm{M}$ staging $(\mathrm{p}=0.209)$ with the prognosis of patients. Multivariate survival analysis proved that Rab40b is an independent prognostic factor for HCC. However, the other clinicopathological characteristics of HCC patients and confounding factors might influence the clinical outcome were not collected, which are the limitation of the study. In addition, the expression of Rab40b in HCC is significantly positively correlated with Ki-67 staining, indicating that Rab40b may play an important role in $\mathrm{HCC}$ progression by promoting the proliferation of tumour cells. Then, we examined the expression of Rab40b on the proliferation and migration of HCC cell lines using gene interference. We found that Rab40b knockdown significantly inhibited the proliferation, migration and invasion of HCC cells in vitro. Subsequently, we found that Rab40b knockdown significantly inhibited tumorigenicity in a nude mouse model.

In addition, we found that Rab40b knockdown induces cell cycle arrest at the G0/G1 phase and increases apoptosis. It has been reported that both cyclin kinase and kinase inhibitors may be involved in the G1/S transition. ${ }^{23,24}$ Cyclin D1 and cyclin E1 are widely regarded as oncogenes and participate in many activities. ${ }^{27,28}$ Conversely, p21 is generally considered a negative regulator of $\mathrm{G} 1 / \mathrm{S}$ transition. ${ }^{25,26}$ Therefore, we determined the expression level of these cell cycle-related proteins by Western blotting. Our results indicated that the downregulation of 
A

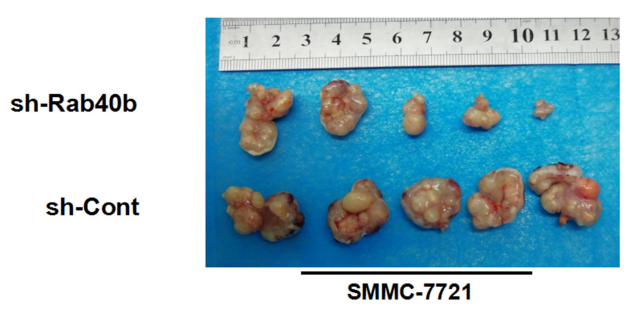

D

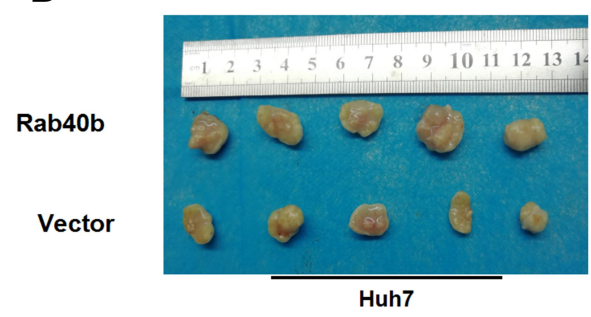

B

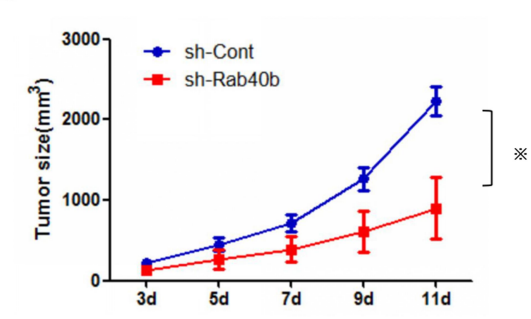

E

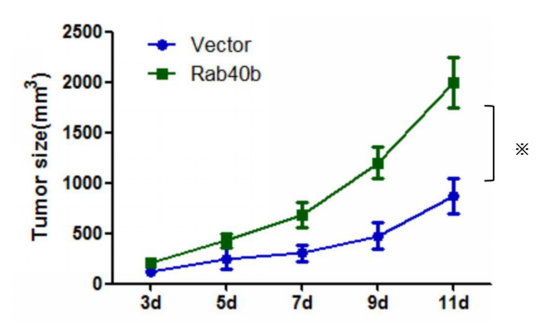

C

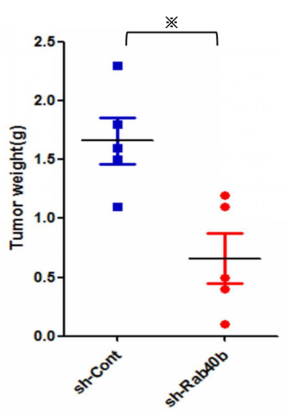

$\mathbf{F}$

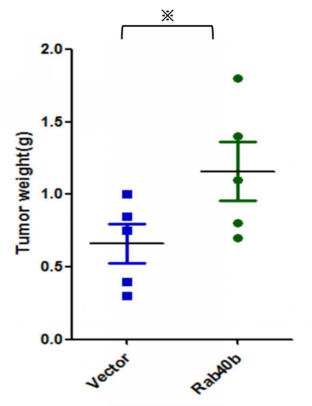

G

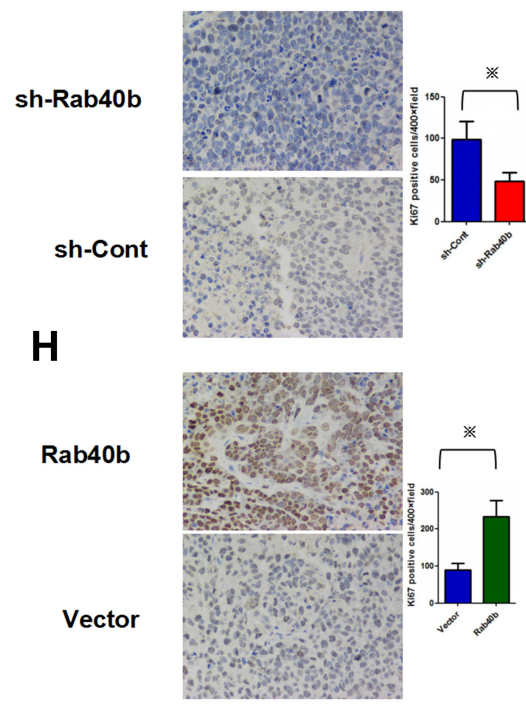

cyclinD1
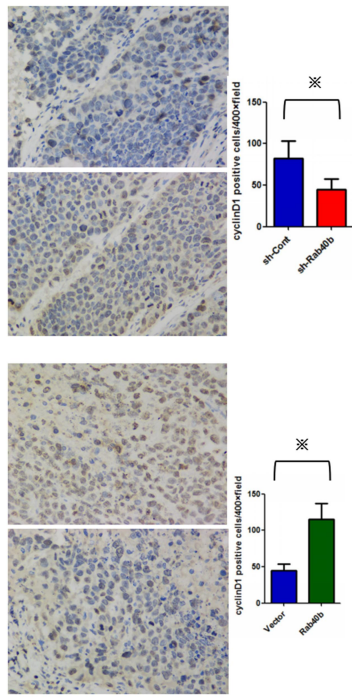

cyclinE1
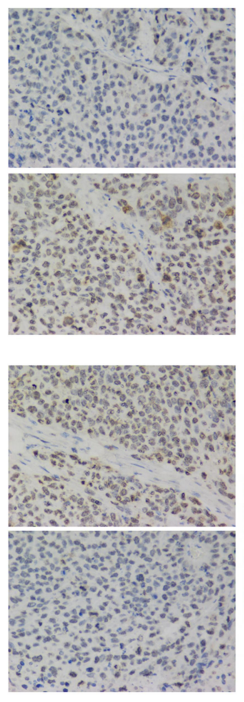

MMP2
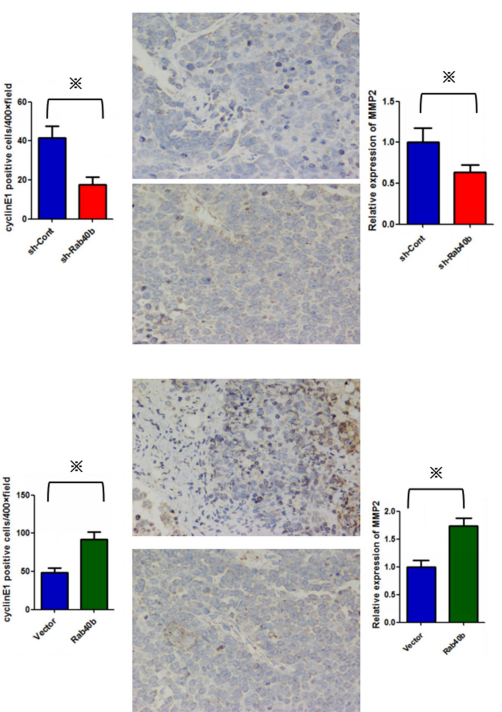

Figure 4 Overexpression of Rab40b promoted the development of xenografts in BALB/c mice. (A) Representative images of tumor size in Rab40b knockdown nude mice model. (B) The tumor size were calculated on each measured time point in Rab40b knockdown model (every two days after xenograft establishment, ${ }^{*}$ < 0.05 . (C) Summary data of tumor weight are provided in Rab40b knockdown model, ${ }^{*} \mathrm{p}<0.05$. (D) Representative images of tumor size in Rab40b overexpressed nude mice model. (E) The tumor size were calculated on each measured time point in Rab40b overexpressed model, ${ }^{*} \mathrm{p}<0.05$. (F) The tumor weight is represented of each group in Rab40b overexpressed model,*p < 0.05. (G) Representative immunohistochemical staining of Ki67, cyclin DI, cyclin El and MMP2 in Rab40b knockdown model,*p < 0.05. (H) Representative immunohistochemical staining of Ki67, cyclin DI, cyclin EI and MMP2 in Rab40b overexpressed model,*p $<0.05$.

cyclin D1 and cyclin E1 and the upregulation of p21 may induce G0/G1 cell cycle arrest in HCC cells after Rab40b knockdown. To further understand the mechanism, we examined the expression of key proteins in cell cyclerelated signalling pathways. PI3K/AKT is recognized as a critical mediator of cell cycle regulation that can participate in the progression of tumours by regulating cell proliferation and apoptosis. ${ }^{29,30} \mathrm{p} 21$, cyclin D1, and cyclin E1 are common downstream regulatory genes of the PI3K/ AKT signalling pathway in a variety of tumours. ${ }^{31,32}$ Therefore, we detected the protein levels of key members of the PI3K/AKT pathway after Rab40b silencing. Western blotting showed that the levels of p-PI3K and p-Akt were significantly reduced by Rab40b knockdown in HCC cells. 


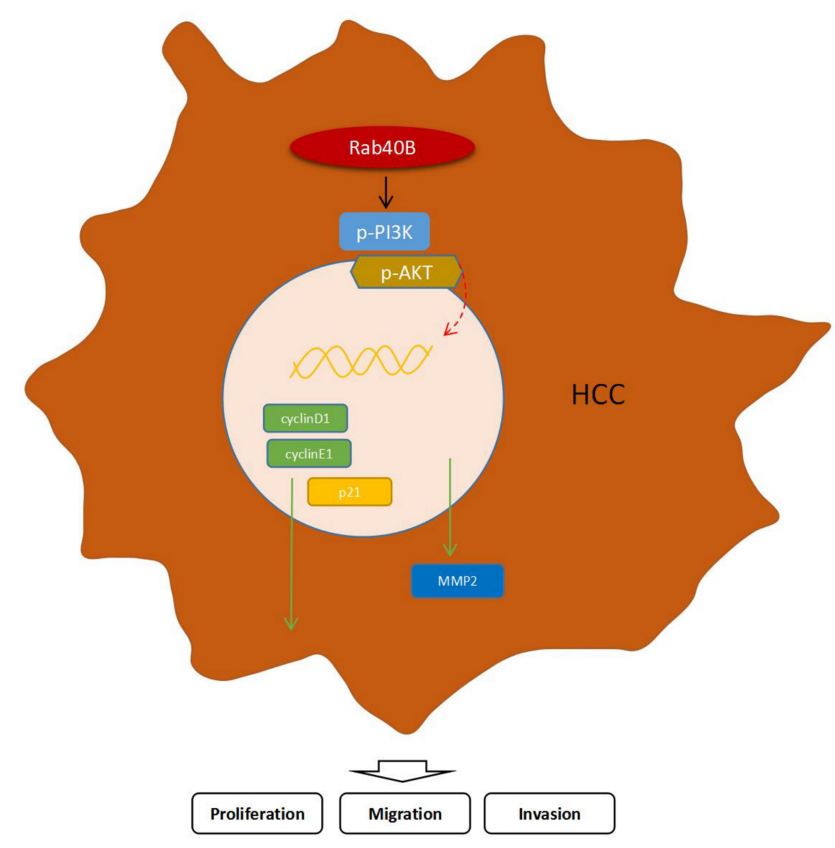

Figure 5 Overexpression of Rab40b promotes hepatocellular carcinoma cell proliferation and metastasis by upregulating cyclin DI, cyclin EI and MMP2 via PI3K/AKT signaling pathway. Overexpression of Rab40b promotes hepatocellular carcinoma cell proliferation by upregulating cyclin DI, cyclin EI and downregulating P2 I via PI3K/AKT signaling pathway. Moreover, overexpression of Rab40b promotes hepatocellular carcinoma cell metastasis by upregulating MMP2 via PI3K/AKT signaling pathway.

Therefore, we hypothesized that Rab40b may promote the downregulation of $\mathrm{p} 21$ and upregulation of cyclin D1 through activation of the PI3K/AKT pathway. However, it is not clear whether Rab40b interacts with other signalling pathways in HCC proliferation. The JAK-STAT pathway is one of the most studied pathways in cell signal transduction and plays a variety of roles in physiological processes. $^{33}$ In addition, we found that caspase-3, which is associated with cell apoptosis, was upregulated after Rab40b knockdown. Therefore, the exact regulation between Rab40b and cleaved caspase-3 needs further investigation. In the future, we will further study the regulatory relationship between Rab40b and the JAK-STAT pathway in HCC progression. Moreover, we further explored the potential mechanism which Rab40b may promote the metastasis of $\mathrm{HCC}$ cells. It is reported that Rab40b regulates the formation of invadopodia and participates in the process of breast cancer cell metastasis by mediating the transport of MMP2 and MMP9. MMP2 and MMP9 closely related to tumor metastasis are two common downstream regulatory genes of the PI3K/AKT signalling pathway. ${ }^{34}$ So we explored whether Rab40b can promote metastasis of HCC cells by regulating MMP2 and MMP9 via PI3K/AKT signalling pathway. Our results demonstrated that Rab40b knockdown significantly decreased the levels of MMP2, p-PI3K and p-AKT. However, the expression of MMP9 had no change.

In conclusion, we demonstrated that Rab40b is an important regulator of HCC progression and an indicator of HCC patient prognosis. Rab40b overexpression promotes the proliferation, invasion and migration of $\mathrm{HCC}$ cell lines by regulating the expression of cyclin D1, cyclin E1, p21 and MMP2 through the PI3K/AKT signalling pathway (Figure 5). Therefore, this study shows that Rab40b is an important oncoprotein and a promising target for HCC therapy. Moreover, it is well known that BRAF inhibition plays a pivotal role in impacting HCC outcome. ${ }^{35}$ Since Rab40b and PI3K orchestrate relevant downstream signalling in HCC biology, it is tempting to speculate a potential combination of Rab40b and PI3K with BRAF-directed therapy. ${ }^{36}$ Our results may provide new potential therapeutic targets for the treatment of advanced $\mathrm{HCC}$.

\section{Acknowledgments}

This work was supported by the National Natural Science Foundation of China (grant No. X2310).

\section{Disclosure}

The authors report no conflicts of interest for this work. 


\section{References}

1. Craig Amanda J, von Felden J, Teresa G-L, et al. Tumour evolution in hepatocellular carcinoma. Nat Rev Gastroenterol Hepatol. 2020;17:139-152.

2. Sandra R, Jean-Charles N. Advances in molecular classification and precision oncology in hepatocellular carcinoma. $J$ Hepatol. 2020;72:215-229.

3. Tianwei C, Fenfen Y, Yanmei Y, et al. CHML promotes liver cancer metastasis by facilitating Rab14 recycle. Nat Commun. 2019;10:2510.

4. Huijuan Y, Jun H, Huan Z, et al. A Rab GTPase protein FvSec4 is necessary for fumonisin B1 biosynthesis and virulence in Fusarium verticillioides. Curr Genet. 2020;66:205-216. doi:10.1007/s00294019-01013-6

5. Siyang S, Wenshu C, Shurong Z, et al. Small GTPases: structure, biological function and its interaction with nanoparticles. Asian J Pharm Sci. 2019;14:30-39. doi:10.1016/j.ajps.2018.06.004

6. Guadagno NA, Progida C. Rab GTPases: switching to Human Diseases. Cells. 2019;16(8):8.

7. Wei $\mathrm{W}$, Weidong J, Bing $\mathrm{H}$, et al. RAB10 overexpression promotes tumor growth and indicates poor prognosis of hepatocellular carcinoma.Oncotarget. 2017;8:26434-26447. doi:10.18632/ oncotarget. 15507

8. Donghua G, Wenyan Z, Yong F, et al. Overexpression of Rab25 promotes hepatocellular carcinoma cell proliferation and invasion. Tumour Biol. 2016;37:7713-7718. doi:10.1007/s13277-015-4606-5

9. Jacob A, Linklater E, Bayless BA, et al. Rab40b and Tks5 interact at invadopodia during metastasis. J Cell Sci. 2016;129:e2301.

10. Martin Z, Luka B, Sylvia E, et al. Bulk tumour cell migration in lung carcinomas might be more common than epithelial-mesenchymal transition and be differently regulated. BMC Cancer. 2018;18:717. doi:10.1186/s12885-018-4640-y

11. Yuanyuan L, Qingzhu J, Wang Y, et al. Rab40b upregulation correlates with the prognosis of gastric cancer by promoting migration, invasion and metastasis. Med Oncol. 2015;32:126.

12. Jacob A, Jing J, Lee J, et al. Rab40b regulates trafficking of MMP2 and MMP9 during invadopodia formation and invasion of breast cancer cells. $J$ Cell Sci. 2013;126:4647-4658. doi:10.1242 jcs. 126573

13. Shi L, Bin Z, Valdes Juan D, et al. Serum Hepatitis B Virus RNA: a new potential biomarker for chronic Hepatitis B virus infection. Hepatology. 2019;69:1816-1827.

14. Xie L, Juan Y, Ruyi X, et al. Cost-effectiveness of antiviral treatment after resection in hepatitis B virus-related hepatocellular carcinoma patients with compensated cirrhosis. Hepatology. 2018;68:1476-1486. doi:10.1002/hep.29922

15. Waisun WV, Janssen Harry LA. Can we use HCC risk scores to individualize surveillance in chronic hepatitis B infection? J Hepatol. 2015;63:722-732. doi:10.1016/j.jhep.2015.05.019

16. Andreas T. Bioinformatics and database resources in hepatology. J Hepatol. 2015;62:712-719.

17. Gopal Krishnan PD, Golden E, Woodward EA, et al. Rab GTPases: emerging oncogenes and tumor suppressive regulators for the editing of survival pathways in cancer. Cancers (Basel). 2020;12:2.

18. Dong JJ, Liu Y, Hao YK, et al. Expression and correlation of Rab23 with pathological grades in human glioma cells. J Biol Regul Homeost Agents. 2019;33:1885-1889.

19. Xiao Y, Zhengshu L, Yongguo L, et al. Rab5a promotes the migration and invasion of hepatocellular carcinoma by up-regulating $\mathrm{Cdc} 42$. Int J Clin Exp Pathol. 2018;11:224-231.

20. Yanxia S, Xiaoqiang Z, Dongli Z. Rab31 promoted hepatocellular carcinoma (HCC) progression via inhibition of cell apoptosis induced by PI3K/AKT/Bcl-2/BAX pathway. Tumour Biol. 2015;36 (1):8661-8670. doi:10.1007/s13277-015-3626-5
21. Abitha J, Erik L, Bayless Brian A, et al. The role and regulation of Rab40b-Tks5 complex during invadopodia formation and cancer cell invasion. J Cell Sci. 2016;129:4341-4353. doi:10.1242/jcs.193904

22. Guorong L, Coralia L, Jianming Q, et al. Role of miR-204 in the regulation of apoptosis, endoplasmic reticulum stress response, and inflammation in human trabecular meshwork cells. Invest Ophthalmol Vis Sci. 2011;52:2999-3007. doi:10.1167/iovs.10-6708

23. Kiran G, Susan TN, Raman M. 4,4'-Diisothiocyanatostilbene-2,2'disulfonate modulates voltage gated $\mathrm{K}$ current and influences cell cycle arrest in androgen sensitive and insensitive human prostate cancer cell lines. Toxicol Mech Methods. 2020;1-28.

24. Yan W, Liangyan S, Wei-Hong Q, et al. Anticancer activity of sugiol against ovarian cancer cell line SKOV3 involves mitochondrial apoptosis, cell cycle arrest and blocking of the RAF/MEK/ERK signalling pathway. Arch Med Sci. 2020;16:428-435. doi:10.5114/ aoms.2017.71420

25. Junbo W, Liang L, Feng W, et al. P21Clinical and prognostic implications of (WAF1/CIP1) expression in patients with esophageal cancer: a systematic review and meta-analysis. Dis Markers. 2020; 2020:6520259.

26. Yan D, Hai G, Liangjun Z, et al. LINC00152 knock-down suppresses esophageal cancer by EGFR signaling pathway. Open Med (Wars). 2020;15:126-133. doi:10.1515/med-2020-0019

27. Nan Z, Min Z, Zhou W, et al. Activated STAT3 could reduce survival in patients with esophageal squamous cell carcinoma by up-regulating VEGF and cyclin D1 expression. $J$ Cancer. 2020;11:1859-1868. doi:10.7150/jca.38798

28. Maryam MB, Afsane B, Majid K, et al. The prognostic value of cyclin D1 expression in the survival of cancer patients: A meta-analysis. Gene. 2020;728:144283. doi:10.1016/j.gene.2019. 144283

29. Silpa S, Arttatrana P. Relevance of Erk1/2-PI3K/Akt signaling pathway in CEES-induced oxidative stress regulates inflammation and apoptosis in keratinocytes. Cell Biol Toxicol. 2019;35:541-564.

30. Dursun G, Christian T, Christian T, et al. Role of PI3K/Akt and $\mathrm{MEK} / \mathrm{ERK}$ signalling in cAMP/Epac-mediated endothelial barrier stabilisation. Front Physiol. 2019;10:1387. doi:10.3389/fphys. 2019.01387

31. Gang X, Chengxue D. CMTM5 is downregulated and suppresses tumour growth in hepatocellular carcinoma through regulating PI3K-AKT signaling. Cancer. Cell Int. 2017;17:113. doi:10.1186/ s12935-017-0485-8

32. Yun $\mathrm{H}$, Chen L, WuCha $\mathrm{Z}$, et al. Isoliquiritigenin inhibits the proliferation, migration and metastasis of Hep3B cells via suppressing cyclin D1 and PI3K/AKT pathway. Biosci Rep. 2020;40(1): BSR20192727. doi:10.1042/BSR20192727

33. Zhi Z, Huiqing W, Jun W, et al. Silencing of EPCAM suppresses hepatic fibrosis and hepatic stellate cell proliferation in mice with alcoholic hepatitis via the PI3K/Akt/mTOR signaling pathway. Cell Cycle. 2019;18:2239-2254. doi:10.1080/15384101.2019.1642067

34. Zhu Y, Yan L, Wenjia Z, et al. MMP2/3 promote the growth and migration of laryngeal squamous cell carcinoma via PI3K/Akt-NF - $\mathrm{BB}$-mediated epithelial-mesenchymal transformation.. $J$ Cell Physiol. 2019.

35. Brunetti O, Gnoni A, Licchetta A, et al. Predictive and prognostic factors in HCC patients treated with sorafenib. Medicina (Kaunas). 2019;55(10):707. doi:10.3390/medicina55100707

36. Gnoni A, Licchetta A, Memeo R, et al. Role of BRAF in hepatocellular carcinoma: a rationale for future targeted cancer therapies. Medicina (Kaunas). 2019;55(12):754. doi:10.3390/medicina55120 754 


\section{Publish your work in this journal}

Cancer Management and Research is an international, peer-reviewed open access journal focusing on cancer research and the optimal use of preventative and integrated treatment interventions to achieve improved outcomes, enhanced survival and quality of life for the cancer patient.
The manuscript management system is completely online and includes a very quick and fair peer-review system, which is all easy to use. Visit http://www.dovepress.com/testimonials.php to read real quotes from published authors.

Submit your manuscript here: https://www.dovepress.com/cancer-management-and-research-journal 